\title{
Dual Quermassintegral Differences for Intersection Body
}

\author{
Lingzhi Zhao1, Jun Yuan ${ }^{*}$ \\ ${ }^{1}$ School of Mathematics and Information Technology, Nanjing Xiaozhuang University, Nanjing, China \\ ${ }^{2}$ College of Teacher Education, Nanjing Xiaozhuang University, Nanjing, China \\ Email: Izhzhao@163.com,
}

Received 12 August 2014; revised 12 September 2014; accepted 19 September 2014

Copyright @ 2014 by authors and Scientific Research Publishing Inc.

This work is licensed under the Creative Commons Attribution International License (CC BY).

http://creativecommons.org/licenses/by/4.0/

(c) (i) Open Access

\begin{abstract}
In this paper, we introduce the concept of dual quermassintegral differences. Further, we give the dual Brunn-Minkowski inequality and dual Minkowski inequality for dual quermassintegral differences for mixed intersection bodies.
\end{abstract}

\section{Keywords}

Intersection Body, Dual Brunn-Minkowski Inequality, Dual Minkowski Inequality

\section{Introduction}

The projection body was introduced in 1934 by Minkowski [1]. The research on the projection body has attracted much attention. The intersection operator and the class of intersection bodies were introduced in 1988 by Lutwak [2], who found a close connection between those bodies and famous Busemann-Petty problem (See [3]-[6]).

In [2], Lutwak presented the mysterious duality between projection and intersection bodies.

For convex bodies $K$ and $L$, let $\Pi K$ and $\Pi_{1}(K, L)$ denote the projection body of $K$ and mixed projection body of $K$ and $L$, respectively. In [7], Lutwak established the following Brunn-Minkowski inequality for projection body and Minkowski inequality for mixed projection body:

Theorem A. Let $K$ and $L$ be convex bodies in $\mathbb{R}^{n}$. Then

$$
V(\Pi(K+L))^{\frac{1}{n(n-1)}} \geq V(\Pi K)^{\frac{1}{n(n-1)}}+V(\Pi L)^{\frac{1}{n(n-1)}},
$$

with equality if and only if $K$ and $L$ are homothetic.

${ }^{*}$ Corresponding author. 
Theorem B. Let $K$ and $L$ be convex bodies in $\mathbb{R}^{n}$. Then

$$
V\left(\Pi_{1}(K, L)\right)^{n-1} \geq V(\Pi K)^{n-2} V(\Pi L),
$$

with equality if and only if $K$ and $L$ are homothetic.

In [8], Theorem A and Theorem B were extended to volume differences:

Theorem C. Suppose that $K, L$, and $D_{1}$ are convex bodies in $\mathbb{R}^{n}$, and $D_{1} \subset K, D_{2} \subset L, D_{2}$ is a homothetic copy of $D_{1}$. Then

$$
\begin{aligned}
& {\left[V(\Pi(K+L))-V\left(\Pi\left(D_{1}+D_{2}\right)\right)\right]^{\frac{1}{n(n-1)}}} \\
& \geq\left[V(\Pi K)-V\left(\Pi D_{1}\right)\right]^{\frac{1}{n(n-1)}}+\left[V(\Pi L)-V\left(\Pi D_{2}\right)\right]^{\frac{1}{n(n-1)}},
\end{aligned}
$$

with equality if and only if $K$ and $L$ are homothetic and $\left(V(K), V\left(D_{1}\right)\right)=\mu\left(V(L), V\left(D_{2}\right)\right)$, where $\mu$ is a constant.

Theorem D. Suppose that $K, L$, and $D_{1}$ are convex bodies in $\mathbb{R}^{n}$, and $D_{1} \subset K, D_{2} \subset L, D_{2}$ is a homothetic copy of $D_{1}$. Then

$$
\left[V\left(\Pi_{1}(K, L)\right)-V\left(\Pi_{1}\left(D_{1}, D_{2}\right)\right)\right]^{n-1} \geq\left[V(\Pi K)-V\left(\Pi D_{1}\right)\right]^{n-2}\left[V(\Pi L)-V\left(\Pi D_{2}\right)\right],
$$

with equality if and only if $K$ and $L$ are homothetic.

For star bodies $K$ and $L$, let $I K$ and $I_{1}(K, L)$ denote the intersection body of $K$ and mixed intersection body of $K$ and $L$, respectively. In [9], Zhao et al. established the following dual Brunn-Minkowski inequality for intersection body and dual Minkowski inequality for mixed intersection body:

Theorem E. Let $K$ and $L$ be star bodies in $\mathbb{R}^{n}$. Then

$$
V(I(K \tilde{+} L))^{\frac{1}{n(n-1)}} \leq V(I K)^{\frac{1}{n(n-1)}}+V(I L)^{\frac{1}{n(n-1)}},
$$

with equality if and only if $L$ is a dilatate of $K$.

Theorem F. Let $K$ and $L$ be star bodies in $\mathbb{R}^{n}$. Then

$$
V\left(I_{1}(K, L)\right)^{n-1} \leq V(I K)^{n-2} V(I L)
$$

with equality if and only if $L$ is a dilatate of $K$.

In this paper, we shall prove the dual forms of inequalities (1.3) and (1.4) for mixed intersection body. In this work new contributions that illustrate this mysterious duality will be presented. Our main results can be stated as follows:

Theorem 1.1. Let $K, L$ and $D_{1}$ are star bodies in $\mathbb{R}^{n}$ and $K \subseteq D_{1}, L \subseteq D_{2}, D_{2}$ is a dilatation of $D_{1}$. Then

$$
\begin{aligned}
& {\left[V\left(I\left(D_{1} \tilde{+} D_{2}\right)\right)-V(I(K \tilde{+} L))\right]^{\frac{1}{n(n-1)}}} \\
& \geq\left[V\left(I D_{1}\right)-V(I K)\right]^{\frac{1}{n(n-1)}}+\left[V\left(I D_{2}\right)-V(I L)\right]^{\frac{1}{n(n-1)}},
\end{aligned}
$$

with equality if and only if $L$ is a dilatate of $K$ and $\left(V(I K), V\left(I D_{1}\right)\right)=\lambda\left(V(I L), V\left(I D_{2}\right)\right)$, where $\lambda$ is a constant.

Theorem 1.2. Let $K, L$ and $D_{1}$ are star bodies in $\mathbb{R}^{n}$ and $K \subseteq D_{1}, L \subseteq D_{2}, D_{2}$ is a dilatation of $D_{1}$. Then

$$
\left[V\left(I_{1}\left(D_{1}, D_{2}\right)\right)-V\left(I_{1}(K, L)\right)\right]^{n-1} \geq\left[V\left(I D_{1}\right)-V(I K)\right]^{n-2}\left[V\left(I D_{2}\right)-V(I L)\right]
$$

with equality if and only if $L$ is a dilatate of $K$.

Please see the next section for related definitions and notations. 


\section{Definitions and Notations}

In this section, we will recall some basic results for dual quermassintegrals of star bodies. The reader is referred to Gardner [10], Lutwak [2] [11] and Thompson [12] for the Brunn-Minkowski theory with its dual theory.

As usual, let $B_{n}$ denote the unit ball in Euclidean $n$-space, $\mathbb{R}^{n}$. While its boundary is $S^{n-1}$ and its volume is denoted by $k_{n}$. For a compact subset $K$ of $\mathbb{R}^{n}$, with $o \in K$, star-shaped with respect to $o$, the radial function $\rho(K, \cdot): S^{n-1} \rightarrow \mathbb{R}$, is defined by

$$
\rho(K, u)=\rho_{K}(u)=\max \{\lambda: \lambda u \in K\} .
$$

If $\rho(K, \cdot)$ is continuous and positive, $K$ will be called a star body.

Two star bodies $K, L$ are said to be dilatate (of each other) if $\rho(K, u) / \rho(L, u)$ is independent of $u \in S^{n-1}$.

The radial sum of two star bodies $K_{1}, K_{2}$ is defined as the star body $K$ satisfying $\rho_{K}=\rho_{K_{1}}+\rho_{K_{2}}$. This operation will be denoted by $\tilde{+}$, i.e., $K=K_{1} \tilde{+} K_{2}$.

For star bodies $K_{1}, \cdots, K_{n}$, the dual mixed volume $\tilde{V}\left(K_{1}, \cdots, K_{n}\right)$ is defined by (see e.g. [11])

$$
\tilde{V}\left(K_{1}, \cdots, K_{n}\right)=\frac{1}{n} \int_{S^{n-1}} \rho_{K_{1}}(u) \cdots \rho_{K_{n}}(u) \mathrm{d} u .
$$

If $K_{1}=\cdots=K_{n-i}=K$, and $K_{n-i+1}=\cdots=K_{n}=B_{n}$, then the dual mixed volume $\tilde{V}\left(K, n-i ; B_{n}, i\right)$ is called dual $i$-quermassintegral of $K$, and denoted by $\tilde{W}_{i}(K)$ and allow $i \in \mathbb{R}$. It is easily seen that $\tilde{W}_{0}(K)=V(K)$.

Let $M$ and $K$ be star bodies in $\mathbb{R}^{n}$. If $K \subseteq M$, then the dual $i$-quermassintegral difference function of $M$ and $K, D \tilde{w}_{i}(M, K) \quad(i \in R ; i \neq n)$, can be defined by

$$
D \tilde{w}_{i}(M, K)=\tilde{W}_{i}(M)-\tilde{W}_{i}(K) .
$$

If $i=0$ in (2.3), then we get the volume difference of star bodies $M$ and $K$ :

$$
D v(M, K)=V(M)-V(K) .
$$

(See [13] for the concept of the volume difference of two compact domains).

The intersection body of a star body $K, I K$, is the centrally symmetric body whose radial function on $S^{n-1}$ is given by [2]

$$
\rho(I K, u)=v\left(K \cap u^{\perp}\right),
$$

where $v$ is $(n-1)$-dimensional volume.

Let $K_{1}, \cdots, K_{n-1}$ be star bodies in $\mathbb{R}^{n}$. The mixed intersection body $I\left(K_{1}, \cdots, K_{n-1}\right)$ of star bodies $K_{1}, \cdots, K_{n-1}$ is defined by

$$
\rho_{I\left(K_{1}, \cdots, K_{n-1}\right)}(u)=\tilde{v}\left(K_{1} \cap u^{\perp}, \cdots, K_{n-1} \cap u^{\perp}\right)=\frac{1}{n-1} \int_{S^{n-1} \cap u^{\perp}} \rho_{K_{1}}(v) \cdots \rho_{K_{n-1}}(v) \mathrm{d} \lambda_{n-2}(v),
$$

where $\tilde{v}$ is $(n-1)$-dimensional dual mixed volume.

If $K_{1}=\cdots=K_{n-i-1}=K, \quad K_{n-i}=\cdots=K_{n-1}=L$, then $I\left(K_{1}, \cdots, K_{n-1}\right)$ will be denoted as $I_{i}(K, L)$. If $K=B_{n}$, then $I_{i}\left(B_{n}, L\right)$ is called the intersection body of order $i$ of $L$; it will often be written as $I_{i} L$. Specially, $I_{n-1} L=I L$. This term was introduced by Zhang [14].

\section{Inequalities for Dual Quermassintegral Differences}

In this section, we will establish two inequalities for dual quermassintegral differences of star bodies, which are generalizations of Theorem 1.1 and Theorem 1.2 presented in introduction.

Theorem 3.1. Suppose that $K, L$ and $D_{1}$ are star bodies in $\mathbb{R}^{n}, D_{2}$ is a dilatate of $D_{1}$. If $0 \leq i \leq n-1$, $K \subseteq D_{1}, L \subseteq D_{2}$, then

$$
D \tilde{w}_{i}\left(I\left(D_{1} \tilde{+} D_{2}\right), I(K \tilde{+} L)\right)^{\frac{1}{(n-i)(n-1)}} \geq D \tilde{w}_{i}\left(I D_{1}, I K\right)^{\frac{1}{(n-i)(n-1)}}+D \tilde{w}_{i}\left(I D_{2}, I L\right)^{\frac{1}{(n-i)(n-1)}},
$$


with equality if and only if $L$ is a dilatate of $K$ and $\left(\tilde{W}_{i}(I K), \tilde{W}_{i}\left(I D_{1}\right)\right)=\mu\left(\tilde{W}_{i}(I L), \tilde{W}_{i}\left(I D_{2}\right)\right)$, where $\mu$ is a constant.

Obviously, the case $i=0$ in Theorem 3.1 is just Theorem 1.1. Furthermore, taking $D_{1}$ and $D_{2}$ to be two closed balls with radii $r_{1}$ and $r_{2}$ in Theorem 1.1, we infer

Corollary 3.2. Let $R(K)$ and $R(L)$ be the circumradii of star bodies $K$ and L. If $r_{1} \geq R(K), r_{2} \geq R(L)$, then

$$
\left[\left(r_{1}+r_{2}\right)^{n(n-1)} k_{n-1}^{n} k_{n}-I(K \tilde{+} L)\right]^{\frac{1}{n(n-1)}} \geq\left[r_{1}^{n(n-1)} k_{n-1}^{n} k_{n}-V(I K)\right]^{\frac{1}{n(n-1)}}+\left[r_{2}^{n(n-1)} k_{n-1}^{n} k_{n}-V(I L)\right]^{\frac{1}{n(n-1)}},
$$

with equality if and only if $L$ is a dilatate of $K$ and $V(I K) / V(I L)=\left(r_{1} / r_{2}\right)^{n(n-1)}$.

Theorem 3.3. Suppose that $K, L$ and $D_{1}$ are star bodies in $\mathbb{R}^{n}, D_{2}$ is a dilatate of $D_{1}$. If $K \subseteq D_{1}, L \subseteq D_{2}$, and $0 \leq i, j<n-1$. Then

$$
D \tilde{w}_{i}\left(I_{j}\left(D_{1}, D_{2}\right), I_{j}(K, L)\right)^{n-1} \geq D \tilde{w}_{i}\left(I D_{1}, I K\right)^{n-j-1} D \tilde{w}_{i}\left(I D_{2}, I L\right)^{j},
$$

with equality if and only if $L$ is a dilatate of $K$ and $\left(\tilde{W}_{i}(I K), \tilde{W}_{i}\left(I D_{1}\right)\right)=\mu\left(\tilde{W}_{i}(I L), \tilde{W}_{i}\left(I D_{2}\right)\right)$, where $\mu$ is a constant.

Obviously, the case $i=0, j=1$ in Theorem 3.3 is just Theorem 1.2.

We will require some additional notations and two analytic inequalities to prove Theorem 3.1 and Theorem 3.3. Firstly, we define a function $\Phi_{p}(x)$ by

$$
\Phi_{p}(x)=\left(x_{1}^{p}-\sum_{i=2}^{n} x_{i}^{p}\right)^{\frac{1}{p}}, x \in \mathbb{R}_{p},
$$

where $\mathbb{R}_{p}=\left\{x=\left(x_{1}, \cdots, x_{n}\right) \mid x_{i} \geq 0, x_{1}^{p} \geq \sum_{i=2}^{n} x_{i}^{p}\right\}$ for $p>0$. Note that $\Phi_{p}^{p}$ is an indefinite form of its variables.

Lemma 3.4. If $p>1, x, y \in \mathbb{R}_{p}$, then $x+y \in \mathbb{R}_{p}$, and

$$
\Phi_{p}(x+y) \geq \Phi_{p}(x)+\Phi_{p}(y),
$$

with equality holds if and only if the coordinates of $x, y$ are proportional.

A proof of Lemma 3.4 can be found in [15]. The inequality (3.1) was first proved by Bellman [16] and is known as Bellman's inequality.

Lemma 3.5. If $a, b, c, d>0,0<\alpha<1,0<\beta<1$ and $\alpha+\beta=1$. Let $a>b$ and $c>d$, then

$$
a^{\alpha} c^{\beta}-b^{\alpha} d^{\beta} \geq(a-b)^{\alpha}(c-d)^{\beta},
$$

with equality if and only if $a / b=c / d$.

Proof. Consider the following function

$$
f(x)=x^{\alpha} c^{\beta}-(x-b)^{\alpha}(c-d)^{\beta}, x>0 .
$$

Let

$$
f^{\prime}(x)=\alpha c^{\beta} x^{\alpha-1}-\alpha(c-d)^{\beta}(x-b)^{\alpha-1}=0 .
$$

We get $x=b c / d$.

On the other hand, if $x \in\left(0, \frac{b c}{d}\right)$, then $f^{\prime}(x)<0$; if $x \in\left(\frac{b c}{d},+\infty\right)$, then $f^{\prime}(x)>0$, and it follows that

$$
\min _{x>0} f(x)=f\left(\frac{b c}{d}\right)=b^{\alpha} d^{\beta}
$$

This completes the proof.

Lemma 3.6. [15] Let $K, L$ be star bodies in $\mathbb{R}^{n}$. If $0 \leq i<n, 1 \leq j<n-1$, then 


$$
\tilde{W}_{i}(I(K \tilde{+} L))^{\frac{1}{(n-i)(n-1)}} \leq \tilde{W}_{i}(I K)^{\frac{1}{(n-i)(n-1)}}+\tilde{W}_{i}(I L)^{\frac{1}{(n-i)(n-1)}},
$$

and

$$
\tilde{W}_{i}\left(I_{j}(K, L)\right)^{n-1} \leq \tilde{W}_{i}(I K)^{n-j-1} \tilde{W}_{i}(I L)^{j},
$$

with equality if and only if $L$ is a dilatate of $K$.

Proof of Theorem 3.1. For star bodies $K, L$, applying inequality (3.2), we have

$$
\tilde{W}_{i}(I(K \tilde{+} L))^{\frac{1}{(n-i)(n-1)}} \leq \tilde{W}_{i}(I K)^{\frac{1}{(n-i)(n-1)}}+\tilde{W}_{i}(I L)^{\frac{1}{(n-i)(n-1)}},
$$

with equality if and only if $L$ is a dilatate of $K$.

$$
\tilde{W}_{i}\left(I\left(D_{1} \tilde{+} D_{2}\right)\right)^{\frac{1}{(n-i)(n-1)}}=\tilde{W}_{i}\left(I D_{1}\right)^{\frac{1}{(n-i)(n-1)}}+\tilde{W}_{i}\left(I D_{2}\right)^{\frac{1}{(n-i)(n-1)}} .
$$

Since $K \subseteq D_{1}, L \subseteq D_{2}$, we get

$$
\tilde{W}_{i}(I K) \leq \tilde{W}_{i}\left(I D_{1}\right), \quad \tilde{W}_{i}(I L) \leq \tilde{W}_{i}\left(I D_{2}\right)
$$

From (3.4) and (3.5), we obtain that

$$
\begin{aligned}
& D \tilde{W}_{i}\left(I\left(D_{1} \tilde{+} D_{2}\right), I(K \tilde{+} L)\right) \\
& \geq\left(\tilde{W}_{i}\left(I D_{1}\right) \frac{1}{(n-i)(n-1)}+\tilde{W}_{i}\left(I D_{2}\right) \frac{1}{(n-i)(n-1)}\right)^{(n-i)(n-1)} \\
& \quad-\left(\tilde{W}_{i}(I K)^{\frac{1}{(n-i)(n-1)}}+\tilde{W}_{i}(I L)^{\frac{1}{(n-i)(n-1)}}\right)^{(n-i)(n-1)} .
\end{aligned}
$$

Then by Lemma 3.4, we get

$$
\begin{aligned}
& D \tilde{W}_{i}\left(I\left(D_{1} \tilde{+}_{2}\right), I(K \tilde{+} L)\right)^{\frac{1}{(n-i)(n-1)}} \\
& \geq\left(\tilde{W}_{i}\left(I D_{1}\right)-\tilde{W}_{i}(I K)\right)^{\frac{1}{(n-i)(n-1)}}+\left(\tilde{W}_{i}\left(I D_{2}\right)-\tilde{W}_{i}(I L)\right)^{\frac{1}{(n-i)(n-1)}} .
\end{aligned}
$$

Note that the equality holds in (3.4) if and only if $L$ is a dilatate of $K$. By Lemma 3.4 we know that the equality holds in (3.6) if and only if $L$ is a dilatate of $K$. and $\left(\tilde{W}_{i}(I K), \tilde{W}_{i}\left(I D_{1}\right)\right)$ is proportional to $\left(\tilde{W}_{i}(I L), \tilde{W}_{i}\left(I D_{2}\right)\right)$.

This completes the proof.

Proof of Theorem 3.3. Applying inequality (3.3), we have

$$
\tilde{W}_{i}\left(I_{j}(K, L)\right)^{n-1} \leq \tilde{W}_{i}(I K)^{n-j-1} \tilde{W}_{i}(I L)^{j},
$$

with equality if and only if $L$ is a dilatate of $K$.

$$
\tilde{W}_{i}\left(I_{j}\left(D_{1}, D_{2}\right)\right)^{n-1}=\tilde{W}_{i}\left(I D_{1}\right)^{n-j-1} \tilde{W}_{i}\left(I D_{2}\right)^{j} .
$$

Hence, by Lemma 3.5, we obtain that

$$
\begin{aligned}
& D \tilde{W}_{i}\left(I_{j}\left(D_{1}, D_{2}\right), I_{j}(K, L)\right) \\
& \geq \tilde{W}_{i}\left(I D_{1}\right)^{\frac{n-j-1}{n-1}} \tilde{W}_{i}\left(I D_{2}\right)^{\frac{j}{n-1}}-\tilde{W}_{i}(I K)^{\frac{n-j-1}{n-1}} \tilde{W}_{i}(I L)^{\frac{j}{n-1}} \\
& \geq\left(\tilde{W}_{i}\left(I D_{1}\right)-\tilde{W}_{i}(I K)\right)^{\frac{n-j-1}{n-1}}\left(\tilde{W}_{i}\left(I D_{2}\right)-\tilde{W}_{i}(I L)\right)^{\frac{j}{n-1}} .
\end{aligned}
$$

The proof is complete. 


\section{Acknowledgments}

We thank the Editor and the referee for their comments. The research is supported by National Natural Science Foundation of China (11101216), Qing Lan Project and the Nanjing Xiaozhuang University (2010KYQN24, 2010KYYB13).

\section{References}

[1] Bonnesen, T. and Fenchel, W. (1987) Theory of Convex Bodies, BCS Associates, Moscow, ID; German Original: Springer, Berlin, 1934.

[2] Lutwak, E. (1988) Intersection Bodies and Dual Mixed Volumes. Advances in Mathematics, 71, 232-261. http://dx.doi.org/10.1016/0001-8708(88)90077-1

[3] Gardner, R.J. (1994) Intersection Bodies and the Busemann-Petty Problem. Transactions of the American Mathematical Society, 342, 435-445. http://dx.doi.org/10.1090/S0002-9947-1994-1201126-7

[4] Gardner, R.J. (1994) A Positive Answer to the Busemann-Petty Problem in Three Dimensions. Annals of Mathematics, 140, 435-447. http://dx.doi.org/10.2307/2118606

[5] Goodey, P., Lutwak, E. and Weil, W. (1996) Functional Analytic Characterizations of Classes of Convex Bodies. Mathematische Zeitschrift, 222, 363-381. http://dx.doi.org/10.1007/BF02621871

[6] Zhang, G.Y. (1999) A Positive Solution to the Busemann-Petty Problem in $\mathbb{R}^{4}$. Annals of Mathematics, 149 535-543. http://dx.doi.org/10.2307/120974

[7] Lutwak, E. (1993) Inequalities for Mixed Projection Bodies. Transactions of the American Mathematical Society, 339, 901-916. http://dx.doi.org/10.1090/S0002-9947-1993-1124171-8

[8] Zhao, C.J. and Cheung, W.S. (2003) On P-Quermassintegral Differences Function. Proceedings of the Indian Academy of Science, 116, 221-231.

[9] Zhao, C.J. and Leng, G.S. (2005) Brunn-Minkowski Inequality for Mixed Intersection Bodies. Journal of Mathematical Analysis and Applications, 301, 115-123. http://dx.doi.org/10.1016/j.jmaa.2004.07.013

[10] Gardner, R.J. (2006) Geometric Tomography. 2nd Edition, Cambridge University Press, New York. http://dx.doi.org/10.1017/CBO9781107341029

[11] Lutwak, E. (1986) Volume of Mixed Bodies. Transactions of the American Mathematical Society, 294, 487-450. http://dx.doi.org/10.1090/S0002-9947-1986-0825717-3

[12] Thompson, A.C. (1996) Minkowski Geometry. Cambridge University Press, Cambridge. http://dx.doi.org/10.1017/CBO9781107325845

[13] Leng, G.S. (2004) The Brunn-Minkowski Inequality for Volume Differences. Advances in Applied Mathematics, 32, 615-624. http://dx.doi.org/10.1016/S0196-8858(03)00095-2

[14] Zhang, G.Y. (1994) Centered Bodies and Dual Mixed Volumes. Transactions of the American Mathematical Society, 345, 777-801.

[15] Losonczi, L. and Páles, Z. (1997) Inequalities for Indefinite Forms. Journal of Mathematical Analysis and Applications, 205, 148-156. http://dx.doi.org/10.1006/jmaa.1996.5188

[16] Beckenbach, E.F. and Bellman, R. (1961) Inequalities. Springer, Berlin. http://dx.doi.org/10.1007/978-3-642-64971-4 
Scientific Research Publishing (SCIRP) is one of the largest Open Access journal publishers. It is currently publishing more than 200 open access, online, peer-reviewed journals covering a wide range of academic disciplines. SCIRP serves the worldwide academic communities and contributes to the progress and application of science with its publication.

Other selected journals from SCIRP are listed as below. Submit your manuscript to us via either submit@scirp.org or Online Submission Portal.
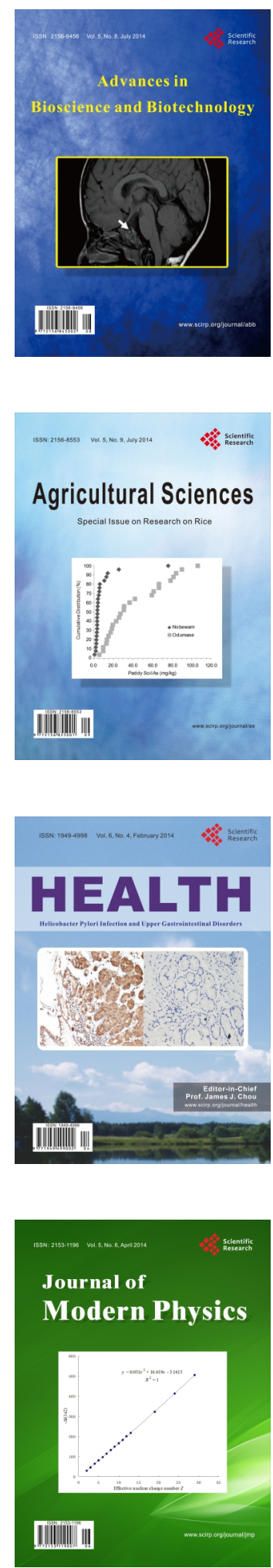
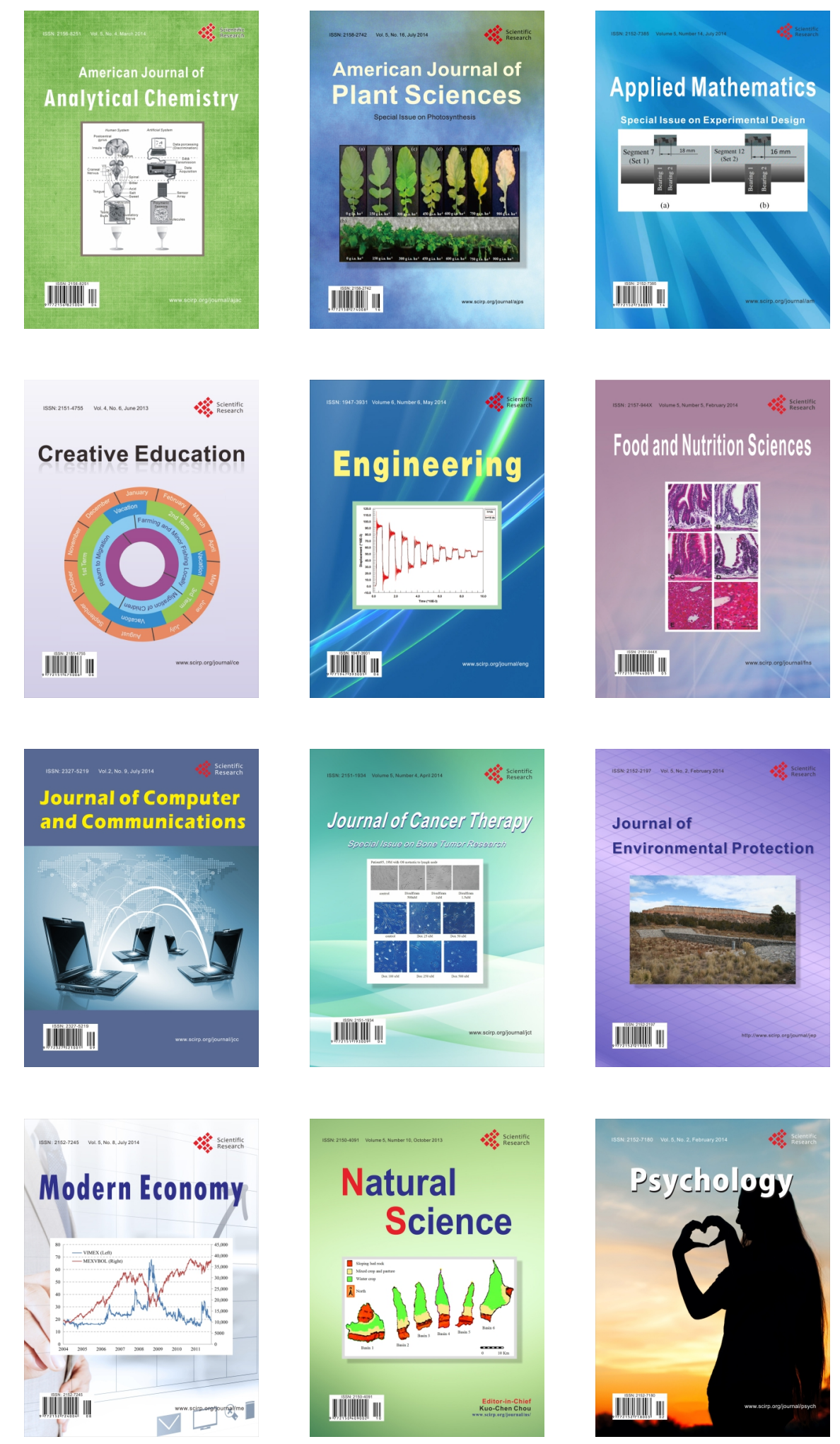